\title{
Taiwan Astroparticle Radiowave Observatory for Geo-synchrotron Emissions (TAROGE)
}

\author{
C.-C. Chen ${ }^{a}$, C.-H. Chen ${ }^{a}$, C.-W Chen ${ }^{a}$, P. Chen ${ }^{a}$, S.-Y. Hsu ${ }^{a}$, J.-J. Huang ${ }^{a}$, M.-H. A. \\ Huang $^{b}$, T.-C. Liu ${ }^{a}$, J.W. Nam ${ }^{a *}$, Y.-S. Shiao ${ }^{a}$, M.-Z. Wang ${ }^{a}$, S.-H.,Wang ${ }^{a}$, T.-L. Zhou ${ }^{a}$ \\ ${ }^{a}$ National Taiwan University, 1 Roosevelt Rd., Taipei, Taiwan \\ ${ }^{b}$ National United University, 2 Lianda Rd., Miao-Li, Taiwan \\ ${ }^{*}$ E-mail: jwnamephys.ntu.edu.tw
}

\begin{abstract}
TAROGE is an antenna array on the high mountains of Taiwan's east coast for the detection of ultra-high energy cosmic ray (UHECR) in an energy round $10^{19} \mathrm{eV}$. The antennas will point toward the ocean to detect radiowave signals emitted by the UHECR-induced air-shower as a result of its interaction with the geomagnetic field. Looking down from the coastal mountain, the effective area is enhanced by collecting both direct-emission as well as the ocean-reflected signals. This instrument also provides the capability of detecting Earth-skimming tau-neutrino through its subsequent tau-decay induced shower. A prototype station with 12 log-periodic dipole array antennas for $110-300 \mathrm{MHz}$ was successfully built at $1000 \mathrm{~m}$ elevation near Heping township, Taiwan in July 2014 to prove the detection concept. It has been operating smoothly for radio survey and optimization of instrumental parameters. We plan to install another station on a higher mountain in summer 2015. In this report, we discuss the design of TAROGE, the performance of the prototype station, and the future prospect.
\end{abstract}

The 34th International Cosmic Ray Conference,

30 July- 6 August, 2015

The Hague, The Netherlands

\footnotetext{
* Speaker.
} 


\section{Introduction}

The radio detection method has recently been established as a promising alternative solution for detecting of Ultra-high energy cosmic rays (UHECRs) which is challenging due to the extremely low flux. It would require a detection area several orders of magnitude larger than the current largest cosmic ray detector; Pierre Auger Observatory [1], to identify their astrophysical sources. Radio emission from extensive air showers is a form of geo-synchrotron radiation, which arises from the deflection of electrons and positions in the shower by the Earth magnetic field. Such radiations at radio-band are coherently added during air-shower development because the radio wavelengths are longer than the shower size, which results in intense pulses that can be detected at a distance of several hundred kilometers. Therefore, one can effectively cover a huge detection area with a small number of radio antennas. Furthermore, the radio detection system can be operated all year round and day-and-night that leads a duty cycle as high as $\sim 100 \%$. Several frontier experiments such as LOPES, ANITA, and AREA $[2,3,4]$ have demonstrated powerful capability of the radio detection method for UHECR detection. For instance, the ANITA experiment, a NASA balloon-borne antenna array that surveys Antarctica from the altitude of $35 \mathrm{~km}$, reported the observation of 16 events with averaged energy $\langle E\rangle=1.5 \times 10^{19} \mathrm{eV}$. It turned out that 14 of these 16 events were radio emissions from down-going UHECRs, but reflected off the Antarctic ice surface. ANITA takes an advantage of vast detection area $\left(\sim 10^{6} \mathrm{~km}^{2}\right)$ within a horizon distance of $\sim 600 \mathrm{~km}$ from the $35 \mathrm{~km}$ of high altitude. Although ANITA has great advantage of covering a large detection area, there are several important drawbacks: 1) the flight duration of the NASA Antarctic balloons is typically limited to $\sim 30$ days. The duty factor further reduces to 10 days/year since the flight opportunity for such balloon projects is roughly once every three years, 2) ANITA's frequency range is $200-1200 \mathrm{MHz}$, which is optimized for UHE neutrino detection $[5,6]$. In contrast, UHE cosmic ray induced geo-synchrotron radiation is significantly enhanced at lower frequencies down to $10 \mathrm{MHz}[7,8,9,10,11]$. Deployment of low frequency antennas to the ANITA payload is difficult due to its weight and space constraints, 3) becuase the strength of EM signal is proportional to $1 / \mathrm{R}$, when it propagates through a long distance to the high altitude, only very high energy events can survive and thus lowers event rate, 4) typical altitude of the shower maximum for UHECRs is $10 \mathrm{~km}$, which is much lower than the ANITA's observation point. Only reflected-off signals are detectable except a small fraction of events coming from near the horizon.

\section{The TAROGE Experiment}

Our approach, Taiwan Astroparticle Radiowave Observatory for Geo-synchrotron Emissions (TAROGE), is to invoke the proven ANITA detection methodology, but place the antennas at the top of high mountains near the ocean. This approach resolves all problems of ANITA described above. Thanks to the ideal geology of Taiwan's east coast, where there are numerous mountains as high as 2 to $3 \mathrm{~km}$ in altitude near ocean. At $2 \mathrm{~km}$ altitude the horizon-distance is $160 \mathrm{~km}$, which would provide a $1.3 \times 10^{4} \mathrm{~km}^{2}$ detection area with a $60^{\circ}$ field-of-view. While losing the detection area relative to ANITA, TAROGE has a great advantage of year-round operation with a high duty cycle $(\sim 90 \%)$, which compensates the area loss. Furthermore, lowering the detection frequency range to $100 \mathrm{MHz}$ (and possibly below) and reducing the detection altitude to $2 \mathrm{~km}$ would enhance 


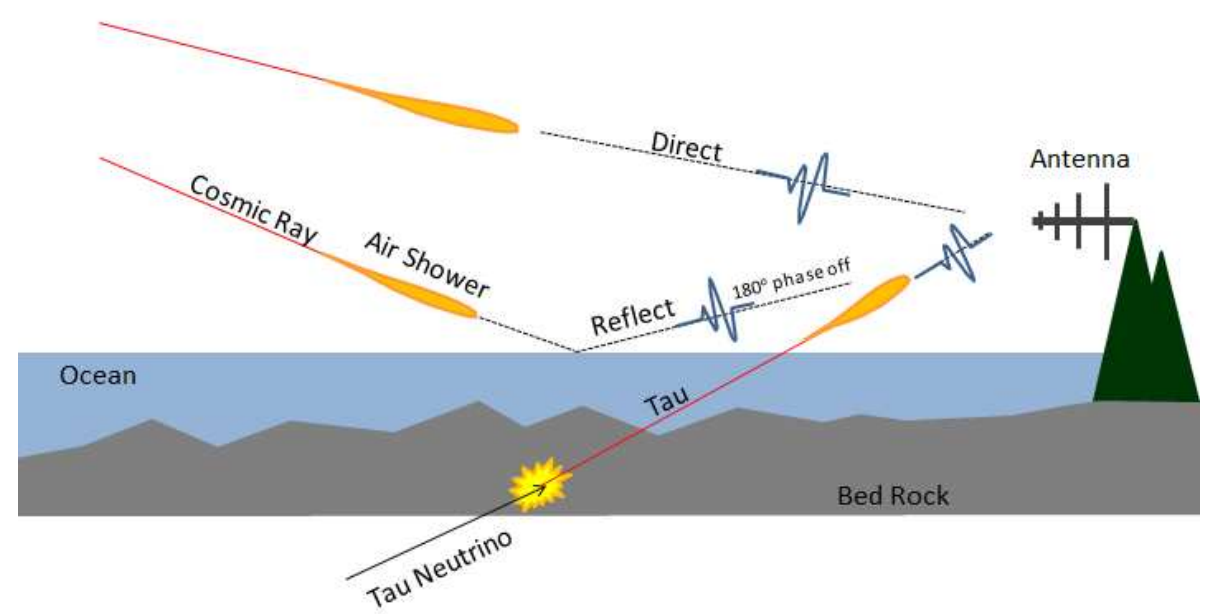

Figure 1: The TAROGE-1 Station; Mechanical rendering (left) and figure after the installation completed (right)

the signal strength and would therefore allow TAROGE to lower the energy threshold. The sea water is highly reflective for Radio Frequency (RF); the reflection coefficient is near $100 \%$ for the horizontally polarized wave, and higher than $60 \%$ for the vertically polarized wave except a very small grazing angle. Surface roughness due to ocean wave would be an issue weakening the signal strength. However, this effect is reduced for low frequency range in which the wavelength is longer than the surface roughness for the typical wave condition. TAROGE's receiver configuration which points toward the horizon is sensitive to showers with large zenith angles. Therefore one can realize that TAROGE is utilized for UHECRS with energies above $10^{18.5}-10^{19} \mathrm{eV}$. The east side of Taiwan is much less populated than other areas of Taiwan, providing a quieter ambient RF environment. Figure 2 shows power spectra obtained from our initial RF survey at multiple locations in the mountain area of eastern Taiwan. Although many frequency bands appear to be polluted by continuous waveform $(\mathrm{CW})$ noises, there are still fairly quite bands where the noise floors are comparable to thermal- and Galactic-noises. Using RF filters and a trigger system which is only sensitive to coherent signal, Taiwan could be a feasible place for this experiment. This detection techinique not only can be applyed in Taiwan, but may also be extended to other places with better and quieter RF environments in future.

\section{The TAROGE-1 Station}

As the first step, in order to demonstrate the detection concept of TAROGE and to perform a long-term noise survey, we built a prototype station, TAROGE-1, in Yongshih Mt. at $1000 \mathrm{~m}$ elevation near Heping township, Taiwan in July 2014. This site had an excellent infrastructure including a good accessibility on a paved road, AC power lines, and a $12 \mathrm{~m}$ tall truss tower for mounting antennas. Because of a fact which the site has both views of the open ocean and the township in the field of view, it is almost ideal location for a detailed RF noise study with various and strong anthropogenic backgrounds near the coast as well as cosmic ray signals. Figure 3 shows overall structure of the TAROGE- 1 station. TAROGE- 1 consists of twelve of $5 \mathrm{dBi}$ log-periodic 

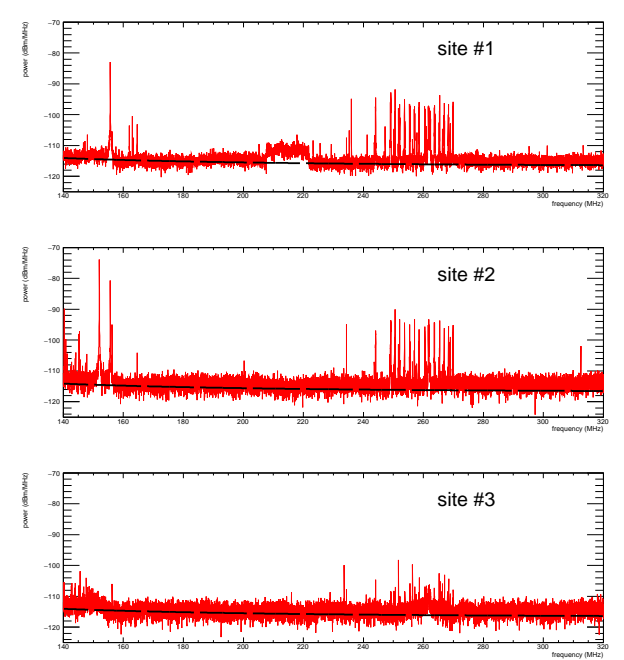

Figure 2: RF noise spectra in the east mountain area: Data taken in a mountain near ocean (top), in a mountain $10 \mathrm{~km}$ far from ocean (middle), and in a deep valey without ocean view (bottom). Dashed lines indicate estimation of the noise floor contributed by thermal- and Galactic-radiations.
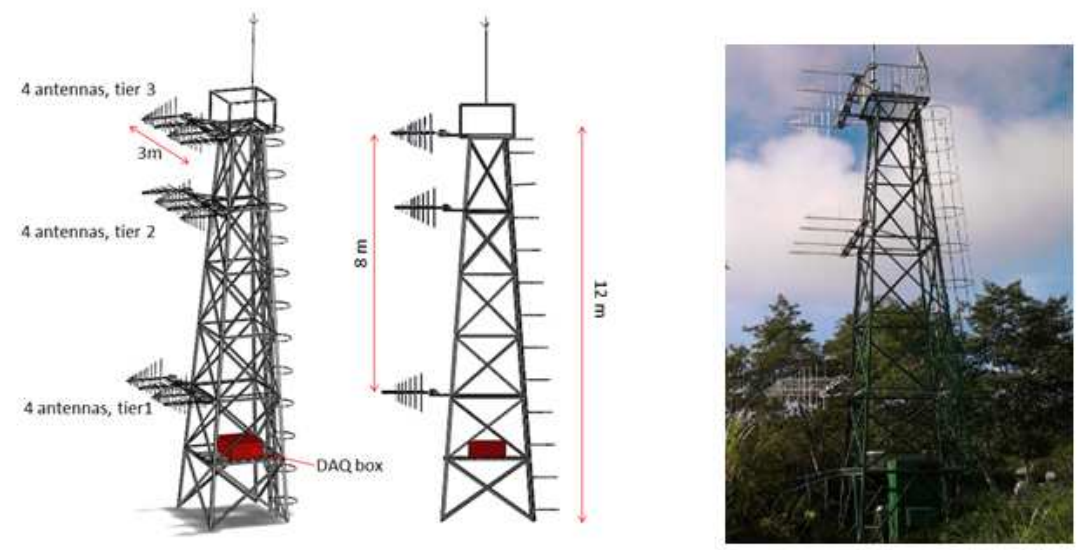

Figure 3: The TAROGE-1 Station; Mechanical rendering (left) and figure after the installation completed (right)

dipole array (LPDA) antennas for 110-300 MHz. A dual polarization configuration, the horizontal polarization (H-pol) and the vertical polarization ( $\mathrm{V}-\mathrm{pol})$, is implemented by orientating E-planes of 6 antennas to the horizontal direction and another to the vertical.

Figure 4 shows the system architecture of TAROGE-1. Received signal in the antenna is fed into the RF-front end before the trigger and digitizer system. The RF front-end consists of a frontend filter, a low noise amplifier $(+35 \mathrm{~dB})$, and a second stage amplifier $(+30 \mathrm{~dB})$. The RF fronts are located close to the LPDAs to minimize transfer losses. Figure 5 shows a schematic diagram of the TAROGE-1 system. The signals are transferred via coaxial cables to the DAQ box where the trigger and digitizer system are placed. In the DAQ box, the RF signals are split into two paths; the trigger and the digitizer. 


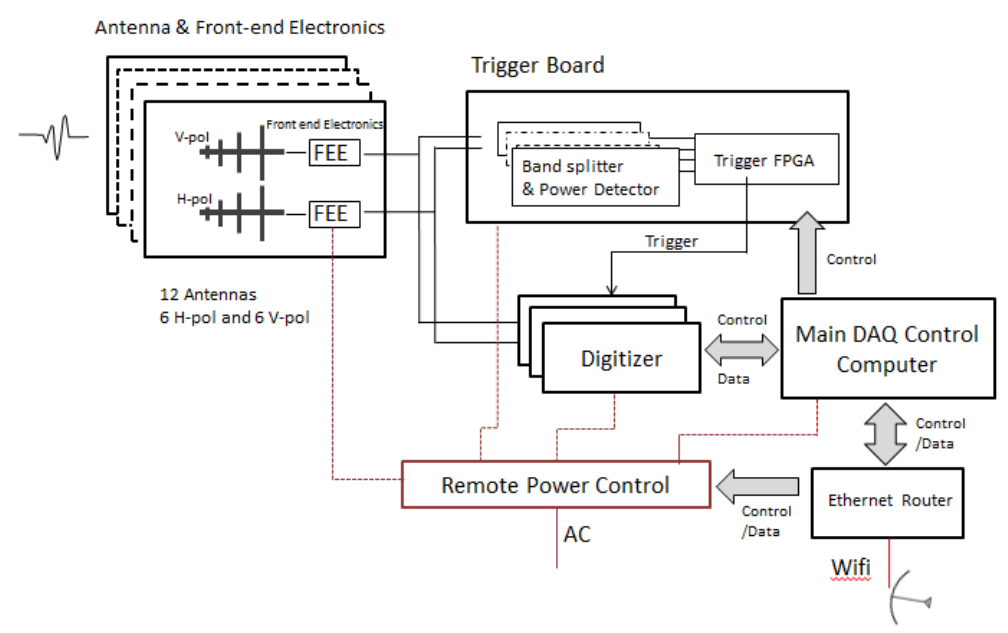

Figure 4: System architecture of TAROGE-1

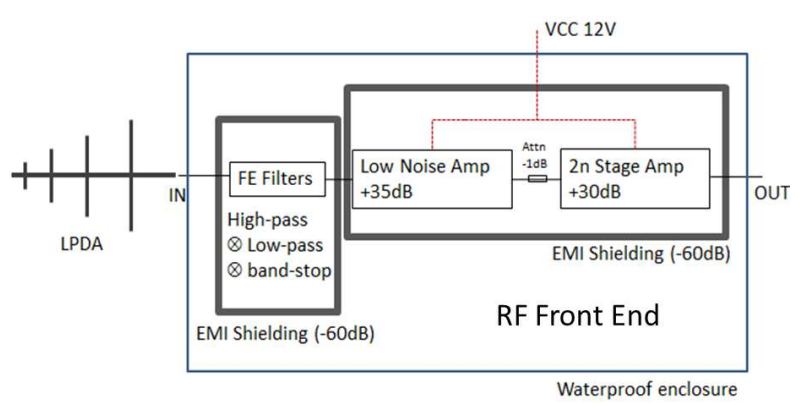

Figure 5: Schematic diagram of RF Front end for TAROGE-1

We use the multi-frequency bands coincidence technique, basically similar to ANITA, which provides an effective discriminating-power for impulsive geo-synchrotron signals against thermal noises as well as $\mathrm{CW}$ anthropogenic-backgrounds. The thermal noise events are further reduced by requiring multiple antennas coincidence. Figure 6 shows architecture of trigger system. The signal path in the trigger system is fed into four bands; $110-140 \mathrm{MHz}, 170-200 \mathrm{MHz}, 220-250 \mathrm{MHz}$, and 270-300 MHz. Uncovered frequency regions between the bands are to avoid communication CW signals along the east coast of Taiwan. Incident increase of RF power is examined by a power detector and a comparator in each band. Individual thresholds of sub-band can be adjusted by multi-channel DACs through remote commands. The thermal noise events are further reduced by requiring multiple antennas coincidence. The dominated emission power of the geo-synchrotron in Taiwan is V-pol because of a low inclination of Earth magnetic field. However, the Askaryan effect in the extensive air-shower still produces a fair amount of power in H-pol [4]. Furthermore, the reflection coefficient of $\mathrm{H}$-pol is much higher than $\mathrm{V}$-pol especially in the low grazing angle which is less than 10 degree. Therefore, we designed the trigger to performe both in H-pol and V-pol, but independently.

The digitizer system uses affordable commercial digital oscilloscopes: GW Instek GDS- 


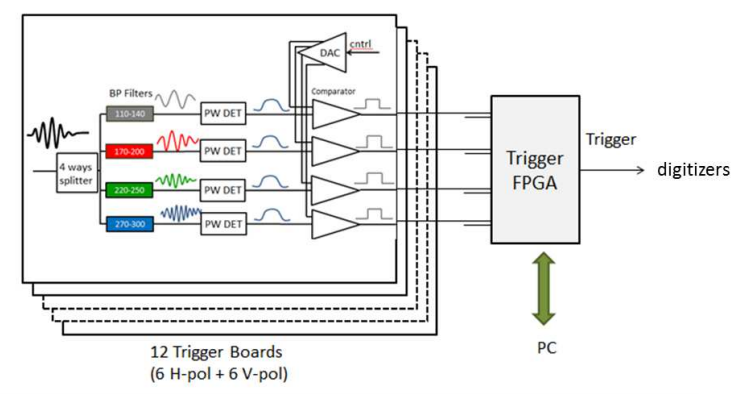

Figure 6: Schematic diagram of trigger system of TAROGE-1

2304A with the $300 \mathrm{MHz}$ bandwidth and 8 bit ADCs. We operate the system with $1 \mathrm{GHz}$ sampling rate for a record length of $1 \mu \mathrm{s}$. The record length is somewhat longer than the optimal configuration, which causes traffic in data transfer to the main computer, but is limited by the company's firmware. The maximum rate of event readout is $\sim 7 \mathrm{~Hz}$, which is the bottleneck of the TAROGE-1 DAQ. The data size is $50 \mathrm{KBytes} / \mathrm{s}$ (or $4.3 \mathrm{GBytes} /$ day) after compression. Real-time data transfer and system control is available through the internet. We use a long range Wi-Fi repeater with 24dBi dish antennas linking between the TAROGE station and the Edu-net at Heping elementary school in the township. All active elements of the system are placed in Faraday boxes to ensure EMI shielding with shielding power better than -60dB. Waterproof enclosures are also used to protect electronics and to prevent change of impedance of RF connectors for severe mountain weather conditions.

A precise geometry survey was carried out to measure coordinates of antennas, which is crucial to ensure a good pointing capability. A laser theodolite with an arc-sec angular resolution was used to provide a precision of $1 \mathrm{~mm}$. After completed the installation of station, system validation and calibration were followed using a calibration pulser system which consists of an impulsive pulse generator and a LPDA antenna. The transmitter power was varied using an adjustable attenuator, and polarization angle was varied by changing tip-tilt angle of an antenna mount on a tripod. The pulser signals were sent from multiple locations near the coastal line where its distance to the TAROGE-1 station is $3-5 \mathrm{~km}$. Because the oscilloscope readout reached the saturation rate due to numerous numbers of impulsive noises from the township, triggering on the pulser events was challenging unless a high threshold signal-to-noise ratio $(S N R>10)$ was required.

Figure 7 displays recorded waveforms of one of the pulser events. The timing calibration was performed using the pulser events. We used the cross-correlation technique [16] to measure arrival time difference between antennas. As shown in Figure 8, we achieved an excellent timing resolution $\sim 80$ ps for $S N R=10$ signals for antenna pairs connected to the same oscilloscope. The antenna pairs in different oscilloscopes has $\sim 400$ ps time resolution which is dominated by the trigger jitter of the oscilloscope. This jitter, in principle, can be removed if the oscilloscopes are synchronized. Several synchronization methods have been proposed, for instance to use the phase information of external sinusoidal waves which added into RF signals before the digitizer. The frequency of this synchronization signal can be chosen out of the TAROGE bands.

We use the interferometry imaging method [17] to determine the arrival direction of the incoming RF signal. Figure 9 shows an interferometry image obtained from the calibration pulser event. 

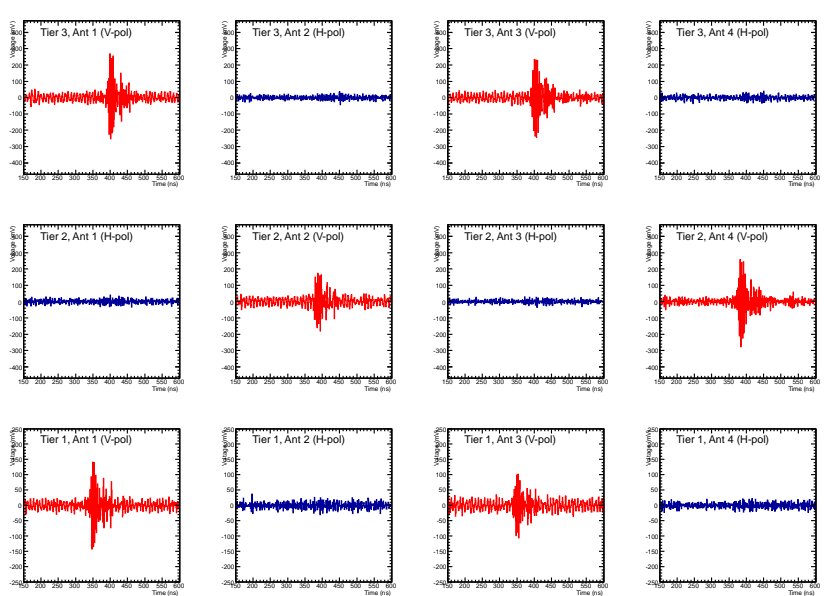

Figure 7: Pulser event waveforms (V-pol event)
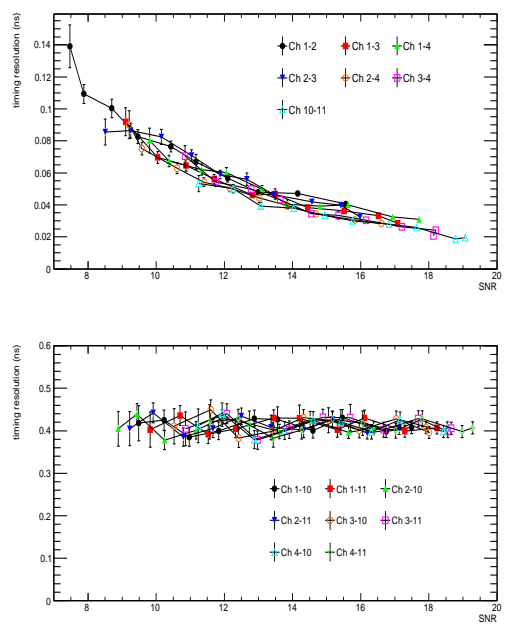

Figure 8: Time resolution versus SNR: channels in the same board (top) and channels in different boards

Figure 10 shows an excellent angular resolutions, which is better than 0.15 degree in elevation angle and 0.45 degree for horizontal angle, are achieved.

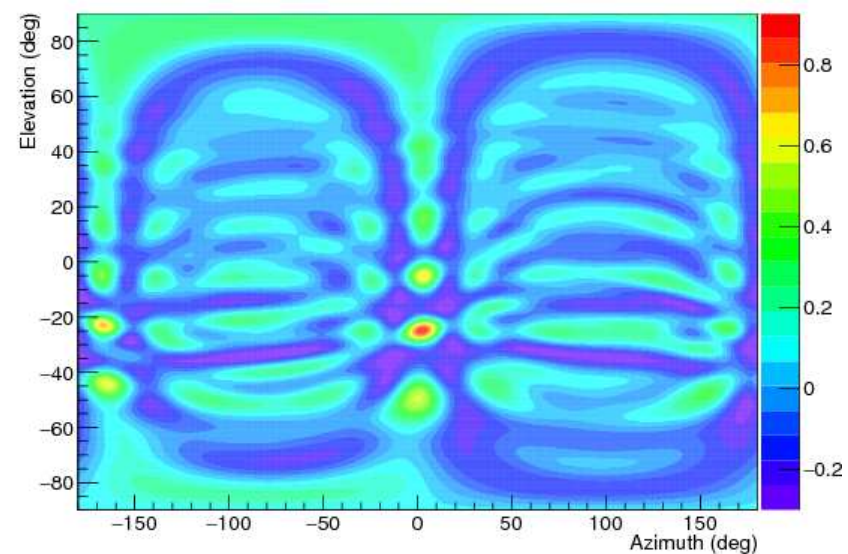

Figure 9: Interferometry image of the calibration pulser event

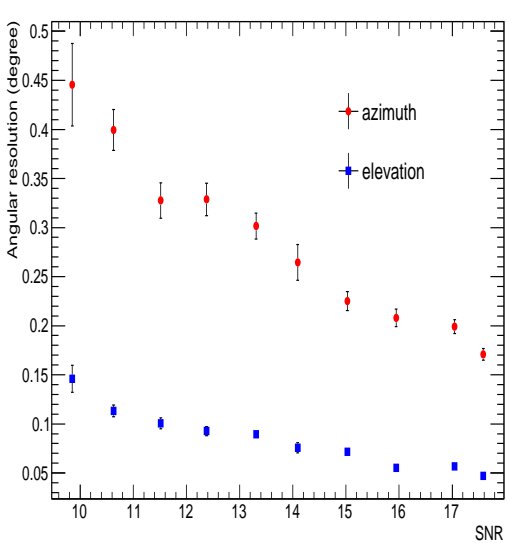

Figure 10: Angular resolution versus SNR

TAROGE-1 has been operating smoothly upto now since the beginning of August 2014. There are some non-operating periods during severe weather conditions as like typhoons, power outages due to failure of power line, and maintanences and minor upgrades of the system. Overall operation time is more than 7 months during past 11 months. The system operated remarkably stable with a duty cycle almost $100 \%$ during winter and spring.

\section{Discussions}

Several important lessons from TAROGE-1 are to be addressed both for system upgrades and 
extensions in future. As we described earlier, operating the trigger system in a low level of threshold are restricted by the severe impulsive noises near the TAROGE- 1 site. The noise sources are mostly identified to be in the township. As an upgrade plan, we are going to install veto antennas pointing toward the township. High gain horn antenna would be a good option for the veto in order to minimize the loss of the detection area. The LPDA antenna design can also be modified for the higher gain. Increasing the boomlength of LPDA by $50 \%$ can provide a gain of $7 \mathrm{dBi}$, which helps reduce the antenna response to the township direction, and also increase the sensitivity to the signals. An inclined orientation of the boresight of LPDA toward the sky by 10-20 degrees can be considered. For the TAROGE-2 station, we found a new site where impulsive noises from the township are drastically suppressed. However, the direction to Hualian which is the biggest city in the east coast and has a local airport, is only 60 degrees off from the middle of the field of view of the new site. Since CW noises from Hualian now become an issue, a new trigger system must be developed not sensitive to the $\mathrm{CW}$ noises. For instance, increasing the number of subfrequency bands would be a feasible solution. The readout rate of the digitizer is to be improved. TAROGE-2 is going to use a new oscilloscope which has a factor of ten higher readout rate and a higher bandwidth $(350 \mathrm{MHz})$ than TAROGE-1. With these improvements, we are going to build the TAROGE-2 station in October 2015 aiming to begin observation of UHECR events.

This work has been supported by the pioneer program of Ministry of Science and Technology, Taiwan. We gratefully thank Taiwan Cement Corporation and Heping elementary school for their invaluable support.

\section{References}

[1] J. Abraham et al., Phys. Lett. B 685 239-246 (2010)

[2] H. Falcke et al., Nature, May 19 issue (2005)

[3] S. Hoover et al., Phys. Rev. Lett.105, 151101 (2010)

[4] T. Huege et al, Nucl. Instr. and Meth. A: 617 484-487 (2010)

[5] P. Gorham et al., Phy. Rev. Lett. 99, 171101 (2007)

[6] P. Gorham et al., Phy. Rev. Lett. 103, 051103 (2009)

[7] T. Huege and H. Falcke, Astropart. Phys. 24, 116-136 (2005)

[8] M. Ludwig and T. Huege, Astropart. Phys. 34, 483-446 (2011)

[9] O. Scholten, K. Werner, and f. Rusydi, Astropart. Phys., 29, 94-103 (2008)

[10] J. Alvarez-Muñiz, W.R. Carvalho, et al., Phys. Rev. D, 86, 123007 (2012)

[11] T. Huege, M. Ludwig, and C.W. James, arXiv:1301.2132 (2013)

[12] D. Heck, et al., FZKA Report 6019, Forschungszentrum Karlsruhe (1998)

[13] T. Huege, R. Ulrich, and R. Engel, Astropart. Physics 27, 392âĂŞ405 (2007)

[14] G. A. Askaryan, JETP 14, 441 (1962)

[15] G. A. Askaryan, JETP 21, 658.(1965)

[16] ANITA Col.: P. Gorham et al., Astroparticle Physics, 32, 10-41 (2010)

[17] A. Romero-Wolf et al., Astroparticle Physics 60, $72-85$ (2015) 